\title{
STRUCTURE OF MULTIVALUED DEPENDENCIES IN RELATION SCHEME
}

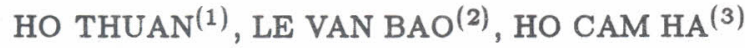

\begin{abstract}
As pointed out in [1], Multivalued dependencies (MVD) depend on the context in which they are defined and thus are very hard to visualize. In this paper, continuing the study in [1], we give some results which may provide some more insight regarding the existence of possible MVDs in the relation schemes provided all their FDs are known in advance.

Throughout this paper, we assume that the reader is familiar with the basic concepts of the relational database $[2,3]$.
\end{abstract}

\section{MULTIVALUED DEPENDENCIES}

Let $U$ be a set attributes, $X$ and $Y$ be disjoint subsets of $U$. We say that in the relation $R$ over the set of attributes $U$, there is a multivalued dependency of the set $Y$ on the set $X$, written $X \rightarrow \rightarrow Y$, if the following holds. Let $Z=U \backslash(X \cup Y)$, a tuple $r$ in $R$ can be viewed as the concatenation of its projection on $X, Y$ and $Z$ which we denote by $r=r[X] r[Y] r[Z]$. Let $r_{1}$ and $r_{2}$ be two tuples with the same $X$-component $r_{1}=r_{1}[X] r_{1}[Y] r_{1}[Z]$ and $r_{2}=r_{1}[X] r_{2}[Y] r_{2}[Z]$ then the interchanged tuples $r_{1}[X] r_{1}[Y] r_{2}[Z]$ and $r_{1}[X] r_{2}[Y] r_{1}[Z]$ are also in $R$. In simple words when $r[X]$ is given, the $Y$-values that appear with it in $r$ are independent of the values of any other attributes. The definition can be generalized to nondisjoint sets $X$ and $Y$. The multivalued dependency $X \rightarrow \rightarrow Y$ holds in $R$ if and only if $X \rightarrow \rightarrow Y \backslash X$ holds in $R$.

We mention here some of the rules that we shall use later:

1. Augmentation axiom:

if $X \rightarrow \rightarrow Y$ and $V \subseteq W$, then $W X \rightarrow \rightarrow V Y$.

2. Coalescence axiom:

if $X \rightarrow \rightarrow Y$ and $V \rightarrow W$ where $W \subseteq Y$ and $Y \cap V=\emptyset$ then $X \rightarrow W$.

3. Mixed pseudotransitivity axiom:

if $X \rightarrow \rightarrow Y$ and $X Y \rightarrow W$ then $X \rightarrow W \backslash Y$.

Let $R$ be a relation scheme, an MVD $X \rightarrow \rightarrow Y$ is said to be nontrivial if $Y \neq \emptyset, Y \nsubseteq X$ and $X Y \neq U$. Since $X \rightarrow \rightarrow Y$ is valid iff $X \rightarrow \rightarrow Y \backslash X$ is valid, we shall always assume that left and right sides of an MVD are disjoint.

Let $Z=U \backslash X Y$, since $X \rightarrow \rightarrow Y$ holds iff $X \rightarrow \rightarrow Z$ holds, we often write the MVD $X \rightarrow \rightarrow Y$ as $X \rightarrow \rightarrow Y \mid Z$. If the FD $X \rightarrow Y$ or the FD $X \rightarrow Z$ is valid, then the MVD $X \rightarrow \rightarrow Y \mid Z$ also holds in $R$. In this case, we call the MVD $X \rightarrow \rightarrow Y \mid Z$, an MVD counterpart of an FD. We call an MVD $X \rightarrow \rightarrow Y \mid Z$ pure if it is nontrivial and it is not an MVD counterpart of an $\mathrm{FD}$ in $R$.

In [1] is has been shown that: 
(a) Let $X \rightarrow \rightarrow Y$ be a nontrivial MVD in $R$ and $K$ any key for $R$, then either $X \rightarrow Y$ holds in $R$ or $K \cap Y \neq \emptyset$.

(b) Let $X \rightarrow \rightarrow Y \mid Z$ be a nontrivial MVD in a relation scheme $R$. Let $K$ be a key for $K$ such that $Y \backslash K \neq \emptyset$. If $Y_{1}=Y \backslash K$ and $Y_{2}=Y \cap K$ then the FD $X Y_{2} \rightarrow Y_{1}$ holds in $R$.

If $X \rightarrow \rightarrow Y \mid Z$ be a pure MVD in a relation scheme $R$. Let $K$ be a key for $R$ such that $Y \backslash K \neq \emptyset$. If $Y_{1}=Y \backslash K$ and $Y_{2}=Y \cap K$ then the FD $X Y_{2} \rightarrow Y_{1}$ hodls in $R$, but the FD $X Y_{2} \rightarrow Z$ does not hold in $R$.

\section{RESULTS}

Theorem 1. Let $K_{1}, K_{2}, \ldots K_{m}$ be the keys for the relation scheme. .

If $Y \cap\left(\bigcap_{i=1}^{m} K_{i}\right)=\emptyset$ and $X \rightarrow \rightarrow Y \backslash K_{i}$ for $i=1,2, \ldots m$ then $X \rightarrow \rightarrow Y$ is not a pure $M V D$.

Proof. By the hypothesis of the theorem it is obviously for all $i=1,2, \ldots m: X \rightarrow \rightarrow Y \backslash K_{i}$ and $K_{i} \cap\left(Y \backslash K_{i}\right)=\emptyset$. Since $K_{i}$ is a key for the relation scheme we have $K_{i} \rightarrow\left(Y \backslash K_{i}\right)$. By the coalescence axiom $X \rightarrow Y \backslash K_{i}$ holds in $R$. From this we have $X \rightarrow \bigcup_{i=1}^{m}\left(Y \backslash K_{i}\right)$.

On the other hand $\bigcup_{i=1}^{m}\left(Y \backslash K_{i}\right)=Y \backslash\left(\bigcap_{i=1}^{m} K_{i}\right)=Y$. Hence $X \rightarrow Y$. The proof is complete.

Remark: Theorem 1 in [1] is a special case of the above theorem with $m=1$.

Corollary 1. Let $X \rightarrow \rightarrow Y$ be a pure $M V D$ in $R$ and let $K_{1}, K_{2}, \ldots K_{m}$ denote the keys for the relation scheme. If $X \rightarrow \rightarrow Y \backslash K_{i}$ for $i=1,2, \ldots$ m then $Y \cap\left(\bigcap_{i=1}^{m} K_{i}\right) \neq \emptyset$.

Theorem 2. Let $X \rightarrow \rightarrow Y$ be a nontrivial $M V D$ in a relation scheme $R$ and $K_{1}, K_{2}, \ldots K_{m}$ denote the keys of $R$ such that $Y \backslash K_{i} \neq \emptyset, \forall i=1,2, \ldots n$.

If $Y \cap\left(\bigcap_{i=1}^{m} K_{i}\right)=\emptyset$ and $X \rightarrow \rightarrow Y \cap K_{i}$ for $i=1,2, \ldots m$ then $X \rightarrow \rightarrow Y$ is not a pure MVD.

Proof. Let $Y_{i}^{1}=Y \backslash K_{i}, Y_{i}^{2}=Y \cap K_{i}$ and $Z=U \backslash X Y$.

From (b) we have $X Y_{i}^{2} \rightarrow Y_{i}^{1}$ holds in $R$. Using $X \rightarrow \rightarrow Y_{i}^{2}$ and $X Y_{i}^{2} \rightarrow Y_{i}^{1}$ we have $X \rightarrow Y_{i}^{1} \backslash Y_{i}^{2}$ i.e. $X \rightarrow Y \backslash K_{i}$. From this $X \rightarrow \bigcup_{i=1}^{m}\left(Y \backslash K_{i}\right)$. Consequently $X \rightarrow\left(Y \backslash \bigcap_{i=1}^{m} K_{i}\right)$ thus $X \rightarrow Y$. The proof is complete.

Corollary 2. Let $X \rightarrow \rightarrow Y$ be a pure $M V D$ in $R$, and let $K_{1}, K_{2}, \ldots K_{m}$ denote the keys of the relation scheme. If $X \rightarrow \rightarrow\left(Y \backslash \cap K_{i}\right)$ for $i=1,2, \ldots m$ then $Y \cap\left(\bigcap_{i=1}^{m} K_{i}\right) \neq \emptyset$.

Theorem 3. Let $X \rightarrow \rightarrow Y$ be a nontrivial $M V D$ in $R$, and $K_{1}, K_{2}, \ldots K_{m}$ denote the keys of the relation scheme, $Y \backslash K_{i} \neq \emptyset$ for $i=1,2, \ldots \mathrm{m}$.

Then $X\left(Y \cap K_{i}\right) \rightarrow X\left(Y \cap K_{j}\right)$ for $i \neq j$.

Proof. We have $\left(Y \cap K_{j}\right) \backslash\left(Y \cap K_{i}\right) \subseteq Y \backslash\left(Y \cap K_{i}\right)$, thus $\left(Y \cap K_{j}\right) \backslash\left(Y \cap K_{i}\right) \subseteq Y \backslash K_{i}$.

Taking into account of (b), we get $X\left(Y \cap K_{i}\right) \rightarrow Y \backslash K_{i}$.

Consequently $X\left(Y \cap K_{i}\right) \rightarrow X\left(Y \cap K_{j}\right) \backslash X\left(Y \cap K_{i}\right)$.

On the other hand $X\left(Y \cap K_{i}\right) \rightarrow X\left(Y \cap K_{j}\right) \cap X\left(Y \cap K_{i}\right)$ and 
$X\left(Y \cap K_{j}\right)=\left\{X\left(Y \cap K_{j}\right) \cap X\left(Y \cap K_{i}\right)\right\} \cup\left\{X\left(Y \cap K_{j}\right) \backslash X\left(Y \cap K_{i}\right)\right\}$.

From this we have $X\left(Y \cap K_{i}\right) \rightarrow X\left(Y \cap K_{j}\right)$. The proof is complete.

Theorem 4. Let $X \rightarrow \rightarrow Y$ be a nontrivial $M V D$ in $R, Y \cap K \neq \emptyset$ and $K$ be any key for $R$ then $X(Y \cap K) \rightarrow Y$.

Proof. We have $X(Y \cap K) \rightarrow Y \cap K$. Taking into account of (b) we get $X(Y \cap K) \rightarrow Y \backslash K$. Consequently $X(Y \cap K) \rightarrow Y$.

Lemma 1. Let $X \rightarrow \rightarrow Y \mid Z$ be a pure $M V D$ in $R$. Then for any key $K: K \backslash Y \neq \emptyset, K \backslash Z \neq \emptyset$.

Proof. Assume the contrary that $K \backslash Y=\emptyset$, i.e., $K \subseteq Y$. Then from $X \rightarrow \rightarrow Y$ and $X Y \rightarrow Z$ we have $X \rightarrow Z$; which contradicts the fact that $X \rightarrow \rightarrow Y \mid Z$ is a pure MVD. Thus $K \backslash Y \neq \emptyset$. Similarly, we can prove $K \backslash Z \neq \emptyset$.

Theorem 5. Let $X \rightarrow \rightarrow Y \mid Z$ be a pure $M V D$ in $R$, and $K$ be any key of $R$, then $|K| \geq 3$.

Proof. Since $X \rightarrow \rightarrow Y \mid Z$ be a pure MVD in $R$, we have $X \rightarrow \rightarrow Y$ and $X \rightarrow \rightarrow Z$. Hence by (a) $K \cap Y \neq \emptyset$ and $K \cap Z \neq \emptyset$. Moreover, by Lemma 1: $K \backslash Y \neq \emptyset$ and $K \backslash Z \neq \emptyset$. Combining with $Y \cap Z=\emptyset$ we have $|K| \geq 3$.

Theorem 6. Let $X \rightarrow \rightarrow Y$ be a nontrivial $M V D$. Then $X \rightarrow Y$ iff there exists a key $K$ such that $X \rightarrow Y \cap K$.

Proof. Let $X \rightarrow Y$. Then, of course, $X \rightarrow Y \cap K$ where $K$ is any key for the relation scheme. Conversely, let $K$ be a key such that $X \rightarrow Y \cap K(\mathrm{i})$.

From $X \rightarrow Y \cap K$, we have $X \rightarrow \rightarrow Y \cap K$. Combining with $X(Y \cap K) \rightarrow Y \backslash K$ (b), and using the mixed pseudotransitivity axiom, we have $X \rightarrow Y \backslash K$ (ii).

From (i) and (ii) we obtain $X \rightarrow Y$.

\section{REFERENCES}

[1] S. Jajoda, Recognizing multivalued dependencies in Relation Scheme, Computer Journal 29 (5) (1986).

[2] Ho Thuan and Le Van Bao, Some results about keys of relational schemes, Acta Cybernetica, Tom 7, Fasc 1, Szeged (1985).

[3] J.D. Ullman, Principles of Database system, 2nd ed., Computer Science Press, Rockville, Md, 1982.

Tóm tăt. Như đã chì rõ trong [1], các phụ thuộc đa trị (MVD) phụ thuộc vào ngữ cảnh trong đó chúng được xác định và do đó rất khó phát hiện. Trong bài báo này, tiểp tục hướng nghiên cứu trong [1], chúng tôi đưa ra một số kết quả làm sáng tó sự tồn tại của các MVD trong các lược đồ quan hệ với tất cả các phụ thuộc hàm (FD) đã biết trước.

Receïved: January 90,1998

(1) Institute of Information Technology - NCST of Vietnam

(2) Open University of Hanoi

(9) Pedagogical Institute of Hanoi 